\title{
Essay
}

Heidi Müller* und Urs Münch

\section{Trauern in Zeiten von Covid-19: Über den Moment hinaus gedacht}

\author{
Grieving in times of COVID-19: thinking beyond
}

https://doi.org/10.1515/spircare-2020-0070

Vorab online veröffentlicht 9. Juni 2020

Zusammenfassung: Das Thema COVID-19 bestimmt den Alltag. Es gelten zahlreiche von der Politik verhängte Einschränkungen und Verbote zur Eindämmung der Pandemie. Für Hinterbliebene können die geltenden Maßnahmen zahlreiche Fragen und Probleme aufwerfen. Dabei betrachtet dieser Artikel vor allem die Auswirkungen, die schon vor dem Auftreten von COVID-19 zu wenig Beachtung gefunden haben. Hierzu gehören der Einfluss der Medien auf die Belastung Betroffener, die Auswirkungen einer unbedachten Wortwahl auf Hinterbliebene, die fehlende Wahrnehmung für die trauerspezifischen Belastungen der Gesundheitsfachkräfte sowie die mangelnde Berücksichtigung des Themas Trauer in Unternehmen.

Schlüsselwörter: Trauer, Belastungen, Pandemie COVID-19

\begin{abstract}
The topic of COVID-19 determines daily life. Numerous restrictions and limitations have been enacted to limit the spread of the virus. For the bereaved these can raise various questions and problems. This article focuses primarily on the effects that had received little attention even before COVID-19 emerged. These include the media influencing the burden of the bereaved, the impact on mourners of terms used in public discussions, the lack of awareness of the grief-specific burden on health professionals and the disregard of grief as an important topic in companies.
\end{abstract}

Keywords: bereavement, restrictions, pandemic COVID-19

*Korrespondenzautorin: Heidi Müller, Trauerzentrum Frankfurt a.M., E-Mail: heidi.mueller@trauerforschung.de Urs Münch, DRK Kliniken Berlin Westend - Klinik für Allgemeinchirurgie, E-Mail: urs.muench@palliativmedizin.de
Das Thema COVID-19 ist allgegenwärtig. Auf der Straße begegnet man Menschen, die einen Mundschutz tragen. Zweidrittel der Nachrichten handeln ausschließlich von COVID-19, der Krieg in Syrien, die Flüchtlinge und Klimaprobleme scheinen völlig vergessen. Nicht zuletzt drängen sich die Appelle von Politikern in den zahlreichen Werbeblöcken, Sondersendungen und Ansprachen auf, die Menschen mögen aus Rücksichtnahme anderen gegenüber bitte zuhause bleiben. Im Zusammenhang mit den geltenden Einschränkungen und Verboten wird von einem alternativlosen Vorgehen gesprochen. In einem Interview in der Süddeutschen Zeitung macht Julie Zeh uns aber darauf aufmerksam, dass ,alternativlos“ nur ein anderes Wort für „Keine Widerrede“ ist (Heidtmann 2020). Denn eine Wahlmöglichkeit, sprich eine Alternative, wurde den Menschen nie präsentiert. Bis heute fehlt es an einem differenzierten Diskurs, der die Chancen und Risiken der verschiedenen Möglichkeiten des Umgangs mit der Pandemie sorgfältig gegeneinander abwägt.

Nicht zuletzt aus diesem Grund haben kürzlich Wissenschaftler und Experten in einem Thesenpapier angeregt, dass die aktuelle Lage nicht nur aus dem pflegerisch-medizinischen Blickwinkel beurteilt werden dürfe (Schrappe 2020). Ebenso entscheidend sei es, Sozial- und Politikwissenschaftler, Ökonomen, Juristen, Public Health Vertreter sowie Ethiker miteinzubinden. Denn jede Präventionsstrategie berge Gefahren und Probleme, die Virologen und Mediziner nicht allein überblicken können.

Vor diesem Hintergrund möchten wir einige Fragen und Probleme benennen, die sich für Trauernde im $\mathrm{Zu}$ sammenhang mit der Corona Pandemie abzeichnen, aber nur selten Beachtung finden.

\section{Trauer - was ist das?}

Im Jahr 2017 sind in Deutschland 932.272 Menschen gestorben (Statistisches Bundesamt, 2019). Die häufigste Todesursache waren Herz-Kreislauf- sowie Krebserkrankungen. Jede Person hinterlässt konservativ geschätzt im 
Schnitt sechs Angehörige beziehungsweise Freunde. Das sind mehr als 5,5 Millionen Trauernde pro Jahr. Keine geringe Anzahl. Menschen sterben. Junge ebenso wie Alte. Das war schon immer so und das wird auch in Zukunft so bleiben. Weitergedacht heißt das aber auch, dass jeder Mensch im Laufe seines Lebens Verluste erleiden wird. Damit ist nicht nur der Tod einer Person gemeint. Die Verlusterfahrungen können sich auch auf andere Verlustobjekte beziehen (z. B. Verlust von Unbeschwertheit, normalem Alltag, Gesundheit, körperlichen Fähigkeiten, Arbeitsplatz). Die darauffolgende Reaktion wird Trauer genannt. Sie dient der Anpassung an die neue Lebenssituation und wird sehr individuell erlebt. Traurigkeit und Sehnsucht können ebenso dazu gehören wie Appetitlosigkeit, Konzentrationsprobleme oder Suchverhalten. Beeinflusst werden die Reaktionsweise und der Verarbeitungsprozess durch zahlreiche Faktoren wie z.B. das soziale Umfeld, die Persönlichkeit oder die finanzielle Situation (Müller et al. 2020a). Doch die Fähigkeit zu trauern, ist biologisch im Menschen angelegt (Archer 2007). Somit können alle Menschen, ganz unabhängig welchen Geschlechts, welcher Nationalität oder beruflichen Fachrichtung, zu Betroffenen werden.

\section{Verlust \& Trauer - omnipräsente Themen}

Nicht erst seit dem Auftreten von COVID-19 sind Sterben, Tod und Trauer omnipräsente Themen. Die Menschen sind fasziniert von allem, was mit dem Tod in Verbindung steht (Penfold-Mounce 2016). Doch in Büchern, Fernsehserien oder Filmen machen andere diese Erfahrung, das eigene Sicherheitsgefühl bleibt unangetastet. Mit jedem Bericht über die COVID-19 bedingten Todesfälle rückt die Möglichkeit der eigenen Betroffenheit allerdings stetig näher und lässt sich immer weniger verdrängen. Dies löst zahlreiche Ängste aus. Dazu gehören neben der Angst, selbst zu sterben oder Angehörige und Freunde zu verlieren, auch die Angst davor, ein überfordertes Gesundheitssystem in Anspruch nehmen und leiden $\mathrm{zu}$ müssen, sowie die Angst davor, alleine zu sterben, Angst davor, kein würdevolles Begräbnis zu erhalten, Angst davor, den Arbeitsplatz und die eigene Existenz zu verlieren, aber auch Angst davor, stärker als bisher Misshandlungen ausgesetzt $\mathrm{zu}$ sein oder, wie möglicherweise bei Politikern, Angst davor, die „falsche“ Entscheidung zu treffen mit allen weitreichenden Konsequenzen für die Gesellschaft, aber auch für die nächste Wiederwahl. Doch was macht Angst mit Menschen? Sie verändert vor allem die Wahrnehmung und die
Informationsverarbeitung. Gefahren werden unter Angst deutlich überschätzt, die Umgebung nach möglichen Gefahren abgescannt. Bedrohliche, stark negative Worte treten in den Vordergrund und verstärken die Angst (Isermann 2016). Das verhindert Besonnenheit und fördert Hysterie. Bei einigen entsteht auch der Wunsch nach Führungspersönlichkeiten, die Sicherheit und einfache Antworten auf komplexe Probleme versprechen. Diese Auswirkungen scheinen vielen Journalisten nicht bewusst $\mathrm{zu}$ sein oder die Entscheidungsträger der Medien nehmen sie billigend in Kauf. Anders lässt sich nicht erklären, wie derzeit über die aktuelle Lage berichtet wird. Die Themen Sterben, Tod und Trauer sind präsenter denn je und werden viel persönlicher genommen als vorher, doch den Medien scheint es in erster Linie um den Wettbewerb der besseren Schlagzeile, der Aufmerksamkeit, der Quote und der Klicks zu gehen.

Erleiden Menschen in einem aufgeheizten, auf Sensationen ausgerichteten medialen Klima einen Verlust und stirbt jemand sogar an den Folgen einer COVID-19 Erkrankung, stellt diese Form der Berichterstattung einen Faktor dar, der die Verarbeitung beeinträchtigen kann. Das haben Untersuchungen zum Beispiel zu den Anschlägen vom 11. September (Silver et al. 2013) oder auf der Insel Utoya (Kristensen et al. 2016) sowie dem Boston Marathon (Holman et al. 2014) gezeigt. Das Auftreten von Depressionen, Angststörungen, aber beispielsweise auch einer Prolonged Grief Disorder werden unter solchen Voraussetzungen wahrscheinlicher. Die Medien tragen also durchaus eine gesellschaftliche Verantwortung. Wenn die Verantwortlichen der Medien um der Quote willen ihrer Verpflichtung zu Sensibilität gegenüber Trauernden nicht nachkommen, ist es für Betroffene derzeit umso wichtiger, sich selbst zu schützen, indem sie ihren Nachrichtenkonsum soweit einschränken, dass er sie nicht belastet

\section{Eine Frage der Wortwahl}

Welch ein Unterschied ein paar Wochen machen. War es vor einiger Zeit noch möglich, in einem Restaurant essen zu gehen, sich mit Freunden zu treffen oder ganz selbstverständlich zur Arbeit zu fahren, gelten mittlerweile zahlreiche Einschränkungen und Verbote, die dazu dienen sollen, die Verbreitung von Covid-19 einzudämmen. Die aktuellen Stichworte lauten „Soziale Distanzierung“, „Abstand halten“ oder auch „soziale Isolation“. Durch zahlreiche Studien wissen wir, dass Einsamkeit und ,Allein Sein“ vielfältige negative gesundheitliche Folgen mit sich bringen können wie z. B. auch eine erhöhte Mortalität (Hawkley \& Cacioppo 2010). Nicht umsonst wird im Strafvollzug die 
Absonderung bzw. Einzelhaft, laienhaft auch Isolationshaft genannt, als besonders schwere Maßnahme gegenüber Straftätern angewandt. Sicher hatten Politiker und ihre Berater nur das Beste für die Bevölkerung im Sinne, als sie die drastischen Einschränkungen und Verbote verhängten. Dennoch gehen damit Konsequenzen einher, die nicht nur gut abgewogen, sondern auch sprachlich adäquat vermittelt werden sollten. Und das auch unter Berücksichtigung dessen, wie Menschen unter Angst Informationen verarbeiten.

Dies scheint derzeit nicht zu gelingen. So können die aktuell gewählten Begriffe eher zusätzliche Belastungen verursachen (Hoy \& Harris 2020). Das gilt insbesondere für Menschen, die einen Verlust erlitten haben und trauern. Das soziale Umfeld ist von großer Bedeutung in Verlustzeiten (Lobb et al. 2010). Angehörige und Freunde leisten lebenspraktische Hilfe, aber auch emotionalen Beistand. Sie sind die wesentliche Stütze bei der Verlustverarbeitung. Wird nun aber von sozialer Distanzierung gesprochen, kann das irreführende Gedanken hervorrufen, die der Idee einer sozialen Isolierung entsprechen. Zusätzlicher Stress bei Betroffenen und das Gefühl von Handlungsunfähigkeit können die Folge sein. Aus diesem Grund ist die Betonung des „körperlichen“ Abstandhaltens oder der Ausdruck „körperliche Distanzierung“ die geeignetere und hilfreichere Wortwahl (Hoy \& Harris 2020). Kontakt ja und unbedingt, aber bitte körperlich Abstand halten. Das ließe Handlungsspielräume für Betroffene zu wie z.B. die Kontaktaufnahme über Computer oder Mobiltelefone und reduzierte somit den Stress, der durch die aktuellen Maßnahmen entstehen kann. Selbstverständlich gilt es zu bedenken, wie man auch jenen Menschen helfen kann, die keinen Zugang zur elektronischen Kommunikation haben. Hier können Telefongespräche und das Briefeschrieben, aber auch nachbarschaftliche Hilfsangebote geeignete Maßnahmen sein. Denn diese Personen sind möglicherweise tatsächlich isoliert und hilfsbedürftig

\section{Fachkräfte in den Blick nehmen}

Aktuell werden nicht nur Ärzte, sondern auch Pflegekräfte als Helden gefeiert. Sie werden besungen, beklatscht und selbst Bonuszahlungen werden Pflegekräften von Politikern in Aussicht gestellt. Da mutet es schon fast zynisch an, dass sie vor dem Hintergrund der Profitmaximierung vor noch nicht allzu langer Zeit hart um Mindestbesetzungen von Stationen, Ausgleichsregelungen und eine zumindest einigermaßen adäquate Bezahlung streiten mussten. Nun gelten sie als Helden, wobei der Drehbuchautor und
Produzent Ralf Husmann in einem Interview mit der Süddeutschen Zeitung zu Recht fragt, ob die neuen Helden denn wirklich eine Alternative haben? Könnten diese Fachkräfte einfach ihren Job kündigen und heimgehen, wenn sie nicht mehr weiterarbeiten möchten (Pollmer 2020)? Endlich erhalten sie die Anerkennung, die ihnen zusteht, möchte man meinen. Doch welchen Preis werden sie dafür zahlen?

Fachkräfte in Kliniken, Alten- und Pflegeheimen oder hospizlichen Einrichtungen sind wie nur wenige andere Berufsgruppen mit Sterben, Tod und Trauer konfrontiert. Dabei haben Todesfälle für die pflegerischen und medizinischen Fachkräfte stets eine Herausforderung dargestellt (Sinclair et al. 2017). So haben Ärzte Schwierigkeiten, ihre Trauer zu verbalisieren und sich Hilfe zu holen, was zu Substanzmissbrauch und Suizid führen kann (Baranowsky \& Schmidt 2013). Palliative-hospizliche Pflegekräfte kontrollieren hingegen ihre Emotionen stark, was zu Burnout und Compassion fatigue führen kann (Barnett et al. 2019). Beide Gruppen leiden darüber hinaus häufig unter dem, was Kenneth Doka „disenfranchised grief“ nennt, also einer geringen Anerkennung der erlebten Verluste (Lamers 2002). Sollten sie nun aufgrund der Corona Pandemie in weit höherem Maße mit Sterben, Tod, Trauer und in dem Zusammenhang auch mit ihrer eigenen Ohnmacht und Hilflosigkeit konfrontiert sein, ist es wichtig, sie viel stärker als bisher nicht nur als besonders stark belastete sondern auch als trauernde Personengruppe in den Blick zu nehmen und ihnen die notwendige Zeit und professionelle Unterstützung zukommen zu lassen, damit sie ihre Verluste verarbeiten können. Zusätzlich gilt es zu bedenken, dass einige Fachkräfte ohnehin hohen Belastungen ausgesetzt sind, etwa weil sie große Sorgen haben, Patienten anzustecken. Das Miterleben von Sterben, Tod und Trauer könnte insbesondere diese Fachkräfte enorm belasten. Das vor allem auch deswegen, weil sie selbst oder ihre nächsten Bezugspersonen im Privatleben potenziell Betroffene sein können und deshalb eine Abgrenzung mit deutlich höherem Kraftaufwand verbunden ist, wenn sie denn überhaupt gelingt.

\section{Trauer am Arbeitsplatz}

Viele kleine und mittelständische Unternehmen sowie große Konzerne haben die Beschäftigung derzeit auf Kurzarbeit umgestellt. Gleichzeitig wird von einer drohenden Rezession gesprochen. Das generiert Existenzängste. Trauernde werden vieles hinnehmen, um einer drohenden Arbeitslosigkeit zu entgehen. Dazu kann es auch gehören, krank zur Arbeit zu gehen oder vollen Arbeitseinsatz zu 
zeigen, obwohl sie sich gerade durch den Verlust belastet fühlen. Fraglich ist, welche Auswirkungen dies auf das Arbeitsumfeld und das Unternehmen hat.

Darüber hinaus könnten Unternehmen mit einer erhöhten Anzahl an Mitarbeitern konfrontiert sein, die gerade einen Verlust erlitten haben. Denkbar ist auch, dass Mitarbeiter oder Kollegen verstorben sind oder versterben werden. Das stellt die Unternehmen vor eine zusätzliche Herausforderung. Mehr denn je stehen sie in der Pflicht für ein Betriebsklima zu sorgen, das jenseits von Profit, Funktionalität und Effektivität auch auf Unterstützung und Menschlichkeit setzt

Das Thema Trauer findet in den Unternehmen bisher wenig Beachtung (Manns 2011). Entsprechend unsicher sind viele Betroffene, wenn sie nach einem Verlust wieder an ihren Arbeitsplatz zurückkehren. Doch auch Vorgesetzte und Kollegen wissen häufig nicht, wie sie mit Hinterbliebenen umgehen sollen. Nicht viel anders ist es, wenn ein Mitarbeiter verstorben ist. Auch hier unterschätzen Arbeitgeber häufig, wie sehr der Tod einzelne bzw. mehrere Kollegen oder auch sie selbst belastet (Lattanzi-Licht 2002). Haben Menschen einen Verlust erfahren und kehren sie an ihren Arbeitsplatz zurück, erleben sie nicht selten eine zunehmende Belastung (Tehan \& Thompson 2013). Herrschen Sprachlosigkeit und Druck vor, kann dies auch negative Folgen für das Unternehmen haben, wie etwa zunehmende Fehlzeiten sowie verminderte Leistungsbereitschaft.

In einer Zeit, in der in erhöhtem Maße und teilweise unter traumatischen Umständen Menschen sterben, ist es wichtig, dass auch Unternehmen sich ihrer sozialen Verantwortung bewusst sind. Entsprechend wären trauerspezifische Schulungen, Bereitstellung einer Informationssammlung zum Thema Trauer, aber auch ein intensiver Kontakt zum Vorgesetzten wichtig, damit Erwartungen und Unterstützung zum Wohle aller aufeinander abgestimmt werden können.

\section{Fazit}

Die Auswirkungen, welche die Corona Pandemie auf Trauernde hat, sind zahlreicher Natur. Aktuell fallen insbesondere die Besuchsverbote in Krankenhäusern, Alten- und Pflegeheimen, Bestattungen im allerkleinsten Kreise, fehlende Abschiednahme von Sterbenden in den Blick. Zahlreiche Experten, aber auch wissenschaftliche Fachgesellschaften haben aus diesem Grund entsprechende Empfehlungen und Hinweise erarbeitet, um die negativen Folgen abzufedern (z. B. Müller et al. 2020 b, Münch et al. 2020). Doch es gilt auch, darüber hinaus zu schauen und vor allem jene Bereiche in den Blick zu nehmen, die schon vor dem Auftreten von COVID-19 wenig Beachtung erfahren haben. Denn hier warten ganz neue Herausforderungen, die nur gesamtgesellschaftlich unter Einbeziehung aller wissenschaftlicher Fachgruppen angegangen werden können.

\section{Literatur}

Archer J (2007) Grief from evolutionary perspective. In: Stroebe MS, Hansson RO, Stroebe W, Schut H (Hg.) Handbook of bereavement research. Consequences, Coping, and care. 5. Aufl., Washington, DC: American Psychological Association. 263-283.

Baranowsky A, Schmidt D (2013) Overcopers: Medical doctor vulnerability to compassion fatigue. In: Figley C, Huggard P, Rees C (Hg.) First do no self-harm: Understanding and promoting physician stress resilience. New York: Oxford University Press. 203-215.

Barnett MD, Hays KN, Cantu C (2019) Compassion fatigue, emotional labor, and emotional display among hospice nurses. DOI: 10.1080/07481187.2019.1699201. [Epub ahead of print].

Hawkley LC, Cacioppo JT (2010) Loneliness matters: a theoretical and empirical review of consequences and mechanisms. Annals of Behavioral Medicine (2):218-227.

Heidtmann J (2020) Es gibt immer eine Alternative. Die Autorin und Verfassungsrichterin Juli Zeh über Handy-Tracking, Denunzianten und die Politik der Angst. Süddeutsche Zeitung 80 (Ausgabe 4./5. April):17.

Holman EA, Garfin DR, Silver RC (2014) Media's role in broadcasting acute stress following the Boston Marathon bombings. Proceedings of the National Academy of Sciences of the United States of America 111(1):93-98.

Hoy WG, Harris HW (2020) Unintended consequences of COVID-19. Grief Perspectives 19 (3):1-4, abrufbar unter http://www.baylor. edu/medical_humanities/.

Isermann M (2016) Krebs und Stress: Hinweise aus der Psychoneuroimmunologie für therapeutisches Handeln. In: Diegelmann C, Isermann M (Hg.) Ressourcenorientierte Psychoonkologie. Psyche und Körper ermutigen. 3. Aufl. Stuttgart: Kohlhammer. 61-79.

Kristensen P, Dyregrov K, Dyregrov A, Heir T (2016) Media exposure and prolonged grief: A study of bereaved parents and siblings after the 2011 Utøya Island Terror Attack. Psychological Trauma: Theory, Research, Practice, and Policy 8(6):661-667.

Lamers WM Jr. (2002) Disenfranchised grief in caregivers. In: Doka KJ (Hg.) Disenfranchised grief. New directions, challenges, and strategies for practice. Champaign, Illinois: Research Press. 181-196.

Lattanzi-Licht M. (2002) Grief and the workplace: Positive approaches. In: Doka KJ (Hg.) Disenfranchised grief. New directions, challenges, and strategies for practice. Champaign, Illinois: Research Press. 167-180.

Lobb EA, Kristjanson LJ, Aoun SM, Monterosso L, Halkett GKB, Davies A (2010) Predictors of complicated grief: A systematic review of empirical studies. Death Studies 34(8):673-698.

Manns ML (2011) Grief and compassion in the workplace. Journal of Behavioral Studies in Business (Zitierdatum 14.04.2020), abrufbar unter https://www.aabri.com/manuscripts/11858.pdf 
Müller H, Münch U, Albang M, Kiepke-Ziemes S für die AG Psychosoziale und Spirituelle Versorgung (2020a) Definition „Trauer im palliativen Kontext" Positionspapier (online). Deutsche Gesellschaft für Palliativmedizin (Zitierdatum 03.05.2020), abrufbar unter https://www.dgpalliativmedizin.de/images/ Positionspapier_AG_Psychosoz._Spirit._Begleitung_Trauer.pdf.

Müller H, Willmann H, Smeding R, Münch U, Wagner B (2020b) Trauern in Zeiten von COVID-19 (online). Gute Trauer (Zitierdatum 03.05.2020), abrufbar unter www.gute-trauer.de.

Münch U, Müller H, Deffner T, von Schmude A, Kern M, Kiepke-Ziemes $S$, Radbruch L (2020) Empfehlungen zur Unterstützung von belasteten, schwerstkranken, sterbenden und trauernden Menschen in der Corona-Pandemie aus palliativmedizinischer Perspektive (online). Deutsche Gesellschaft für Palliativmedizin (Zitierdatum 03.05.2020), abrufbar unter https://www.dgpallia tivmedizin.de/images/DGP_Unterstuetzung_Belastete_ Schwerstkranke_Sterbende_Trauernde.pdf.

Penfold-Mounce R (2016) Corpses, popular culture and forensic science: Public obsession with death. Mortality 21(1):19-35.

Pollmer C (2020) Ralf Husmann über Helden. Süddeutsche Zeitung 80 (Ausgabe 4./5. April):52.

Schrappe M, François-Kettner H, Gruhl M, Knieps F, Pfaff H, Glaeske G (2020) Thesenpapier zur Pandemie durch SARS-CoV-2/Covid-19.
Datenbasis verbessern Prävention gezielt weiterentwickeln Bürgerrechte wahren (online). (Zitierdatum 12.04.2020), abrufbar unter: https://www.springerpflege.de/resource/con tent/\%22Thesenpapier\%20zur\%20Pandemie\%20...\%22\%20im $\% 20$ Wortlaut.pdf/17868958.

Silver RC, Holman EA, Andersen JP, Poulin M, McIntosh DN, GilRivas V (2013) Mental- and physical-health effects of acute exposure to media images of the September 11, 2001, attacks and the Iraq War. Psychological Science 24:1623-1634.

Sinclair S, Raffin-Bouchal S, Venturato L, Mijovic-Kondejewski J, Smith-MacDonald L (2017) Compassion fatigue: a meta-narrative review of the healthcare literature. International Journal of Nursing Studies 69:9-24.

Statistisches Bundesamt (Destatis) (2019) Todesursachen. Anzahl der Gestorbenen nach Kapiteln der ICD-10 und nach Geschlecht für 2017 (online). Statistisches Bundesamt (Zitierdatum 14.04.2020), abrufbar unter https://www.destatis.de/DE/Them en/Gesellschaft-Umwelt/Gesundheit/Todesursachen/Tabellen/ gestorbene_anzahl.html.

Tehan M, Thompson N (2013) Loss and grief in the workplace: The challenge of leadership. OMEGA - Journal of Death and Dying 66(3):265-280. 